\title{
Une étude de la qualité des blés hybrides à travers différents tests technologiques
}

\author{
FX Oury ${ }^{1 *}, M_{\text {Rousset }}{ }^{1}$, P Bérard ${ }^{1}$, P Pluchard ${ }^{2}$, G Doussinault ${ }^{3}$ \\ avec la collaboration technique de $\mathrm{J}$ Gourdon ${ }^{1}$ et $\mathrm{F}$ Lagoutte ${ }^{1}$ \\ ${ }^{1}$ INRA, station d'amélioration des plantes, domaine de Crouelle, F63039 Clermont-Ferrand cedex; \\ 2 INRA, domaine de Brunehaut, Estrées-en-Chaussées, F80200 Péronne; \\ ${ }^{3}$ INRA, station d'amélioration des plantes, domaine de la Motte, BP29, F35650 Le Rheu, France
}

(Reçu le 31 janvier 1994 ; accepté le 15 juillet 1994)

\begin{abstract}
Résumé - Trente-trois hybrides de blé tendre d'hiver produits par voie chimique ont été comparés à leurs 27 parents pour la qualité. Les échantillons de grains utilisés pour les tests technologiques provenaient d'une expérimentation multilocale, mais les interactions "génotypes x milieux" sont apparues négligeables pour la dureté, le Pelshenke et l'alvéographe Chopin. Aucun hétérosis par rapport au parent supérieur n'a été mis en évidence pour ces 3 tests, ni pour la teneur en protéines : la F1 se situe généralement entre ses 2 parents. Dans le cas de la teneur en protéines, ce résultat semble indiquer une relation «source-puits" plus favorable chez les hybrides que chez les parents, car le taux de protéines est maintenu chez la F1 alors qu'il existe un hétérosis significatif pour le rendement en grains. On observe d'ailleurs un hétérosis significatif pour le rendement en protéines. En définitive, la sélection de blés hybrides de bonne qualité ne semble pas devoir poser de problème majeur.
\end{abstract}

\section{blé hybride / qualité / Pelshenke / alvéographe / dureté}

Summary - Bread-making quality of wheat hybrids through various technological tests. Thirty-three winter wheat hybrids produced using a chemical hybridizing agent, and their 27 parents, were compared for bread-making quality. Samples of kernels used for technological tests were obtained in a multilocal experiment, but the 'genotype $x$ environment' interactions appeared negligible for grain hardness, the Pelshenke test and alveograph measurements. Heterosis did not occur, either for these 3 tests or for the protein content, and the F1 was intermediate between the 2 parents. For protein content, this could indicate a 'source-sink' relationship that is more favourable for hybrids than for inbred lines. This is because the protein content was maintained in spite of a significant heterosis for grain yield. In fact, there was a significant heterosis for protein yield. From all these results, it appears that breeding hybrid wheats with good quality characteristics should not be a major problem.

hybrid wheat / bread-making quality / Pelshenke / alveograph / grain hardness

* Correspondance et tirés à part 


\section{INTRODUCTION}

La complexité des déterminismes génétiques de la productivité et de la qualité et l'influence du milieu sur leur expression font qu'il est difficile de réunir dans un même génotype l'ensemble des caractéristiques permettant d'obtenir à la fois un rendement élevé et une haute valeur technologique. C'est ainsi que les progrès en termes de productivité ont été plus faibles pour les blés de qualité (Bingham et Lupton, 1987). En outre, si la productivité est une variable qui ne pose pas de gros problèmes de définition et de mesure, la qualité, elle, est une variable plus difficile à appréhender. En effet, elle dépend du type de valorisation (panification française, panification anglo-saxonne, biscuiterie, etc), et n'est pas mesurable directement : par exemple, il n'est pas possible de réaliser des panifications sur un ensemble de génotypes en sélection, d'une part pour des raisons de disponibilité en grains, et d'autre part parce que le coût en serait beaucoup trop élevé. L'évaluation de la qualité se fait donc par des tests indirects, qui nécessitent des quantités de grain réduites, mais qui ne renseignent alors que sur certains paramètres de cette qualité.

Les travaux récents sur les blés hybrides (Borghi et al, 1989 ; Morgan et al, 1989 ; Oury et al, 1990, 1993) ont montré que la voie hybride permettait d'améliorer la productivité, grâce à l'existence d'un hétérosis par rapport au parent supérieur pour le rendement. En revanche, les travaux relatifs à la qualité des blés hybrides sont plus rares et, excepté ceux de Perenzin et al (1992), ont porté sur un nombre d'hybrides réduit (Boland et Walcott, 1985 ; Borghi et al, 1988 ; Brears et al, 1988). De plus, l'étude génétique de la qualité est plus difficile dans le cas des blés hybrides. En effet, les grains récoltés sur une $F 1$ présentent 3 types de tissus génétiquement distincts :

- le péricarpe diploïde, qui provient de la plantemère ;

- l'embryon diploïde, qui résulte de la fécondation et correspond à la génération $\mathrm{F} 2$;

- l'albumen triploïde, qui est issu de la fécondation de 2 gamètes femelles identiques par un gamète mâle, et appartient aussi à la génération F2.

Cet article présente les résultats d'une étude réalisée dans le cadre de la collaboration entre I'INRA et le GIE «Hybriblé» (lequel regroupe les établissements de sélection Blondeau,
Hybrinova, Momont et UCASP, et la firme Orsan - pour la recherche sur les agents chimiques d'hybridation). Notre objectif était de situer la valeur technologique de la $\mathrm{F} 1$ par rapport à celle de ses parents, et cela sur un effectif d'hybrides suffisamment important pour que les résultats aient une certaine valeur statistique.

\section{MATÉRIEL ET MÉTHODES}

Les échantillons de grains qui ont été utilisés pour les analyses technologiques provenaient d'essais réalisés en 1991 dans 7 lieux : les stations INRA de ClermontFerrand (CF), Le Moulon ( $L M$ ) et Rennes (RE), les stations d'Hybrinova à Pouy (P), Saint-Germain (SG) et Estrées (E), et la station de l'UCASP à Arles (A). Les techniques culturales appliquées en chacun des lieux correspondaient aux conduites intensives habituellement pratiquées dans la région, avec en particulier des apports N,P, K ajustés à des objectifs de rendement élevés, et une couverture fongicide et insecticide efficace.

Les hybrides étudiés ont été produits avec l'agent chimique d'hybridation d'Orsan, à partir de lignées fournies par le GIE «Hybriblée ou les stations INRA partenaires de ce GIE, et de variétés commerciales. Ces hybrides faisaient intervenir 18 parents femelles et 9 parents mâles, sans plan de croisement particulier. Les 18 parents femelles étaient tous de bonne valeur agronomique, et avaient été choisis de manière à ne pas être apparentés (Oury, 1990). L'apparentement entre les 9 parents mâles n'a pas été étudié, mais ils étaient d'origines diverses et tous de bonne valeur agronomique. Les 27 lignées parentales couvraient une gamme de valeurs technologiques étendue.

Au total, 33 combinaisons hybrides et leurs 27 parents ont été étudiés pour la qualité. Cependant, seule une partie de ces 60 génotypes était présente dans chaque lieu (tableau I). Pour essayer de regrouper les résultats, nous avons étudié les interactions "génotype $x$ milieu», d'une part au moyen de représentations graphiques, et d'autre part en calculant les corrélations inter-lieux pour chacune des variables étudiées, à chaque fois que 2 lieux avaient au moins 5 génotypes en commun.

On ne disposait pas d'échantillons de grains pour les parents d'un des 4 hybrides expérimentés à Pouy, et pour les parents de 7 des 10 hybrides expérimentés à Saint-Germain. Pour ces $8 \mathrm{~F} 1$ la comparaison avec les parents n'a donc pas été possible.

La dureté et la teneur en protéines du grain ont été mesurées par réflectance dans le proche infra-rouge. Les caractéristiques rhéologiques de la pâte ont été évaluées à travers le test Pelshenke et l'alvéographe de Chopin. Ce dernier donne une mesure de la force $(W)$, de la ténacité $(P)$ et de l'extensibilité $(L)$ de la pâte. À partir de $P$ et $L$ on calcule le rapport $P / L$, qui traduit l'équilibre de la courbe alvéographique. Pour 2 parents femelles et les 2 hybrides dans lesquels ces 2 parents intervenaient, l'alvéographe Chopin n'a pas pu 
Tableau I. Effectifs étudiés, valeurs moyennes et niveaux d'hétérosis (en \%) pour le rendement, la teneur en protéines, et le rendement en protéines, dans les 7 implantations.

\begin{tabular}{|c|c|c|c|c|c|c|c|}
\hline & $C F$ & $P$ & $A$ & $R E$ & $L M$ & $S G$ & $E$ \\
\hline \multicolumn{8}{|l|}{ Effectifs } \\
\hline Parents & 12 & 6 & 13 & 13 & 10 & 10 & 9 \\
\hline Hybrides & 11 & 4 & 16 & 16 & 8 & 10 & 6 \\
\hline \multicolumn{8}{|l|}{ Rendement } \\
\hline Moyenne $(q / h a)$ & 88,3 & 97,8 & 79,7 & 92 & 89,2 & 91,9 & 62,8 \\
\hline Hétérosis PM & $+1,2$ & $+6,6\left(^{\star}\right)$ & $+3,6\left(^{*}\right)$ & $+8,8^{\star \star \star}$ & $+8,9^{\star \star}$ & $+11,1^{\star \star}$ & $+7,1^{\star *}$ \\
\hline Hétérosis PS & -5 & $+4,9$ & $-1,9$ & $+4,3^{\star}$ & $+4,8^{\star}$ & $+7,9^{*}$ & $+3,5\left(^{\star}\right)$ \\
\hline \multicolumn{8}{|l|}{ Teneur en protéines } \\
\hline Moyenne (\%) & 13,3 & 12,2 & 11,9 & 12,2 & 13,4 & 12,6 & 12 \\
\hline Hétérosis PM & $-2,4$ & $-3,2$ & $+0,2$ & $+0,1$ & $+0,2$ & +0 & $+2,1^{*}$ \\
\hline Hétérosis PS & $-4,6$ & $-6,7$ & $-2,2$ & $-2,8$ & $-1,8$ & $-5,9$ & $-1,9$ \\
\hline \multicolumn{8}{|c|}{ Rendement en protéines } \\
\hline Moyenne (q/ha) & 11,7 & 11,9 & 9,4 & 11,2 & 11,9 & 11,6 & 7,5 \\
\hline Hétérosis PM & $-1,2$ & $+3,3$ & $+3,6^{\star}$ & $+8,9^{\star \star \star}$ & $+8,9^{\star \star \star}$ & $+10,9^{\star \star}$ & $+10,4^{\star \star}$ \\
\hline Hétérosis PS & -8 & $+0,5$ & $-0,6$ & $+3^{\star}$ & $+5,5^{\star}$ & $+1,4\left(^{\star}\right)$ & $+3,7$ \\
\hline
\end{tabular}

À côté des valeurs d'hétérosis figurent les résultats du test de Student unilatéral permettant de tester la supériorité des hybrides par rapport aux parents moyen et supérieur. (") significatif au seuil de $10 \% ;{ }^{*}$ significatif au seuil de $5 \%$; ${ }^{*}$ significatif au seuil de $1 \%$; ${ }^{* * *}$ significatif au seuil de 1\%. CF : Clermont-Ferrand ; P : Pouy ; A : Arles ; RE : Rennes ; LM : Le Moulon ; SG : Saint-Germain ; E : Estrées. PM : parent moyen ; PS : parent supérieur.

être réalisé. Pour ce test, l'effectif se réduit donc à 31 hybrides et 25 parents.

L'ensemble des traitements graphiques et statistiques a été réalisé à l'aide du logiciel «S" (Becker et al, 1988).

\section{RÉSULTATS ET DISCUSSION}

Les niveaux de rendement obtenus dans les différentes implantations (tableau I) apparaissent satisfaisants, sauf à Estrées où l'essai a souffert de la sécheresse. Les valeurs moyennes de teneur en protéines sont du même ordre de grandeur dans les 7 implantations (tableau I). En particulier, les rendements plus faibles enregistrés à Estrées ne se sont pas traduits par des teneurs en protéines anormalement élevées qui auraient pu introduire un biais dans notre étude de la qualité.

\section{Interactions "génotypes $x$ milieux"}

Les graphiques d'interaction (non présentés) et le tableau II permettent de distinguer les 2 groupes de variables suivants :
- pour le rendement et la teneur en protéines, les classements des génotypes sont très différents selon le lieu, ce qui indique de fortes interactions "génotype $x$ milieu". Pour ces 2 variables, le regroupement des données provenant des différentes implantations n'est pas possible, et nous les avons donc étudiées lieu par lieu ;

- pour la dureté, le Pelshenke et, à un degré moindre, les paramètres de l'alvéographe Chopin, les classements des génotypes apparaissent relativement stables quel que soit le lieu. Pour ces 5 variables, nous avons donc considéré que les interactions «génotypes $x$ milieux» pouvaient être négligées. Nous avons alors procédé à un regroupement des données, en prenant comme valeur unique caractérisant un génotype, la moyenne des valeurs obtenues par ce génotype dans les différentes implantations où il était présent, après correction de l'effet «lieu».

\section{Comparaisons entre les hybrides et leurs parents}

Pour le rendement, il y a généralement un hétérosis par rapport au parent moyen significatif (tableau I), et en moyenne l'hybride se situe au- 
Tableau II. Corrélations de rangs de Spearman pour les différentes variables étudiées.

\begin{tabular}{|c|c|c|c|c|c|c|c|}
\hline & \multirow[t]{2}{*}{ Rendement } & \multirow{2}{*}{$\begin{array}{l}\text { Teneur en } \\
\text { protéines }\end{array}$} & \multirow[t]{2}{*}{ Dureté } & \multirow[t]{2}{*}{ Pelshenke } & \multicolumn{3}{|c|}{ Alvéographe } \\
\hline & & & & & $w$ & $P$ & $L$ \\
\hline CF-A & $\begin{array}{c}-0,21 \\
(9)\end{array}$ & $\begin{array}{c}0,67\left(^{*}\right) \\
(9)\end{array}$ & $\begin{array}{c}0,92^{* * *} \\
(9)\end{array}$ & $\begin{array}{c}0,94^{\star \star \star} \\
(9)\end{array}$ & $\begin{array}{c}0,83^{\star \star} \\
(9)\end{array}$ & $\begin{array}{c}0,74^{*} \\
(9)\end{array}$ & $\begin{array}{c}0,8^{\star \star} \\
(9)\end{array}$ \\
\hline CF-RE & $\begin{array}{c}0,04 \\
(9)\end{array}$ & $\begin{array}{c}0,66\left(^{*}\right) \\
(9)\end{array}$ & $\begin{array}{c}0,95^{\star \star \star} \\
(9)\end{array}$ & $\begin{array}{c}0,96^{\star \star \star} \\
(10)\end{array}$ & $\begin{array}{c}0,82^{\star \star} \\
(9)\end{array}$ & $\begin{array}{c}0,65\left(^{*}\right) \\
(9)\end{array}$ & $\begin{array}{c}0,82^{\star *} \\
(9)\end{array}$ \\
\hline CF-LM & $\begin{array}{l}0,39 \\
(18)\end{array}$ & $\begin{array}{c}0,64^{\star \star} \\
(18)\end{array}$ & $\begin{array}{c}0,96^{\star \star \star} \\
(18)\end{array}$ & $\begin{array}{c}0,96^{\star \star *} \\
(18)\end{array}$ & $\begin{array}{c}0,83^{* * *} \\
(18)\end{array}$ & $\begin{array}{c}0,69^{\star *} \\
(18)\end{array}$ & $\begin{array}{c}0,72^{\star \star \star} \\
(18)\end{array}$ \\
\hline CF-SG & $\begin{array}{l}0,12 \\
(13)\end{array}$ & $\begin{array}{c}0,54\left(^{*}\right) \\
(12)\end{array}$ & $\begin{array}{c}0,94^{\star * *} \\
(12)\end{array}$ & $\begin{array}{c}0,97^{\star * *} \\
(13)\end{array}$ & $\begin{array}{c}0,8\left(^{\star}\right) \\
(5)\end{array}$ & $\begin{array}{c}0,85\left(^{*}\right) \\
(5)\end{array}$ & $\begin{array}{c}0,81\left(^{*}\right) \\
(5)\end{array}$ \\
\hline P-A & $\begin{array}{l}0,05 \\
(10)\end{array}$ & $\begin{array}{l}0,45 \\
(10)\end{array}$ & $\begin{array}{c}0,96^{\star * *} \\
(10)\end{array}$ & $\begin{array}{c}0,96^{\star \star \star} \\
(10)\end{array}$ & $\begin{array}{c}0,86^{\star \star} \\
(8)\end{array}$ & $\begin{array}{l}0,93^{\star \star \star} \\
(8)\end{array}$ & $\begin{array}{c}0,51 \\
(8)\end{array}$ \\
\hline P-RE & $\begin{array}{l}0,42 \\
(10)\end{array}$ & $\begin{array}{l}0,39 \\
(10)\end{array}$ & $\begin{array}{c}0,94^{\star \star *} \\
(10)\end{array}$ & $\begin{array}{c}0,97^{\star \star \star} \\
(10)\end{array}$ & $\begin{array}{c}0,94^{\star \star \star} \\
(8)\end{array}$ & $\begin{array}{c}0,96^{* \star *} \\
(8)\end{array}$ & $\begin{array}{l}0,55 \\
(8)\end{array}$ \\
\hline A-RE & $\begin{array}{c}0,51^{\star *} \\
(29)\end{array}$ & $\begin{array}{c}0,65^{\star \star \star} \\
(29)\end{array}$ & $\begin{array}{c}0,91^{* \star *} \\
(29)\end{array}$ & $\begin{array}{c}0,91^{\star \star \star} \\
(29)\end{array}$ & $\begin{array}{c}0,91^{* * *} \\
(29)\end{array}$ & $\begin{array}{c}0,84^{\star \star \star} \\
(29)\end{array}$ & $\begin{array}{c}0,73^{\star \star *} \\
(29)\end{array}$ \\
\hline LM-SG & $\begin{array}{c}0,65^{*} \\
(13)\end{array}$ & $\begin{array}{l}0,46 \\
(12)\end{array}$ & $\begin{array}{c}0,95^{\star * *} \\
(12)\end{array}$ & $\begin{array}{c}0,96^{\star \star \star} \\
(12)\end{array}$ & $\begin{array}{c}0,97^{\star \star} \\
(5)\end{array}$ & $\begin{array}{c}0,93^{*} \\
(5)\end{array}$ & $\begin{array}{c}0,89^{\star} \\
(5)\end{array}$ \\
\hline
\end{tabular}

La valeur indiquée entre parenthèses correspond au nombre de génotypes communs aux 2 lieux (les seuls couples de lieux considérés sont ceux pour lesquels cette valeur est au moins égale à 5). (") significatif au seuil de $10 \%$; " significatif au seuil de $5 \%$; ${ }^{\star \star}$ significatif au seuil de $1 \% ;{ }^{* \star \star}$ significatif au seuil de $1 \%$.

dessus du parent supérieur (sauf à Clermont et à Arles). La figure 1 montre que les distributions des rendements des hybrides sont décalées vers les fortes valeurs par rapport aux distributions des rendements des parents (sauf à Clermont). On retrouve donc bien la supériorité des $F 1$ pour la productivité (Borghi et al, 1989 ; Morgan et al, 1989 ; Oury et al, 1990, 1993).

Pour la teneur en protéines l'hybride se situe entre ses 2 parents, et les valeurs de l'hétérosis par rapport au parent moyen sont proches de zéro dans les 7 lieux (tableau I). En outre, la figu-

\section{RENDEMENT}

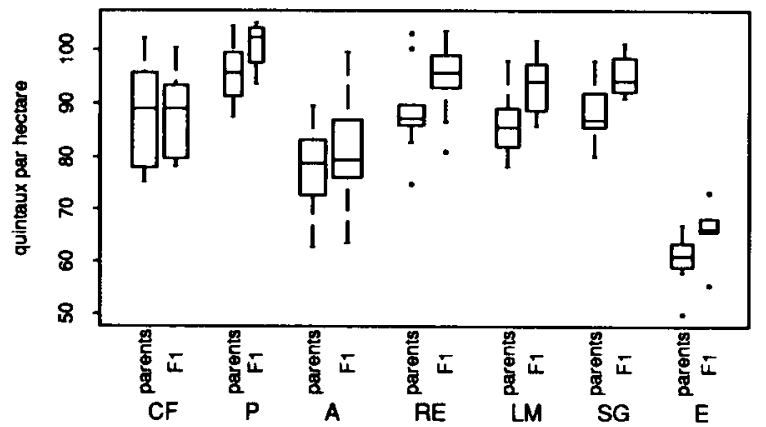

\section{TENEUR EN PROTEINES}

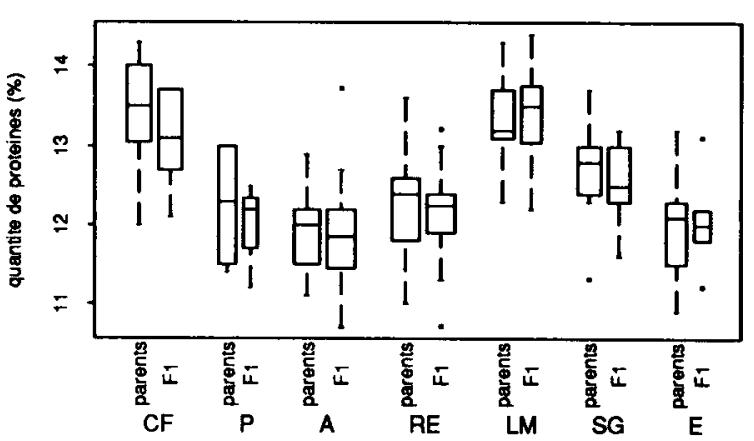

Fig 1. Distributions du rendement et de la teneur en protéines (représentées sous forme de box-plots) pour les parents et les hybrides, dans les 7 lieux. 
re 1 montre que les distributions sont analogues pour les $\mathrm{F} 1$ et leurs parents dans tous les lieux. Ces résultats confirment ceux déjà obtenus par Brears et al (1988) et Perenzin et al (1992).

Plusieurs auteurs (Payne et al, 1987 ; Brunori et al, 1989 ; Carrillo et al, 1990) considèrent qu'il existe une relation négative entre le rendement et la teneur en protéines, même si cette relation n'est pas toujours forte. Dans notre cas, cette relation n'est significative que dans 3 lieux sur 7 : Arles, Rennes, et, dans une moindre mesure, Pouy (tableau III). On constate cependant que le signe du coefficient de corrélation calculé entre les 2 variables est généralement négatif, et que la relation est plus forte pour les $\mathrm{F} 1$ que pour leurs parents (à Estrées le potentiel de rendement n'a pas pu s'exprimer de manière normale, ce qui explique sans doute les coefficients positifs obtenus dans ce lieu). Le fait que la teneur en protéines des hybrides reste comparable à celle des parents, malgré l'expression d'un hétérosis pour le rendement, est donc remarquable. De fait, si on calcule les rendements en protéines, on constate qu'il existe généralement un hétérosis par rapport au parent moyen significatif pour cette variable (tableau I), et qu'en moyenne l'hybride se situe au-dessus du parent supérieur (sauf à Clermont et Arles). Ceci rejoint le résultat rapporté par Boland et Walcott (1985), mais sur un seul hybride. Nous avons montré que, pour l'accumulation de la matière sèche dans le grain (Oury et al, 1993), les hybrides se distinguaient des lignées par une relation «source-puits» plus favorable. Cet avantage de la situation hybride concernerait donc non seulement l'amidon (constituant principal de la matière sèche du grain), mais également la fraction protéique.

Pour la dureté, la figure 2 permet de distinguer 2 lots de génotypes parentaux : ceux qui ont une valeur inférieure à 10 , et ceux qui ont une valeur comprise entre 23 et 42 . Les premiers peuvent être rattachés à la catégorie des blés "soft», et les seconds à celle des blés "medium hard" (Mahaut, 1993). Le déterminisme génétique de la

Tableau III. Corrélations entre la teneur en protéines $(\% \mathrm{~N})$ d'une part, et le rendement et les 5 variables technologiques d'autre part, pour les 7 implantations.

\begin{tabular}{|c|c|c|c|c|c|c|c|}
\hline & $C F$ & $P$ & $A$ & $R E$ & $L M$ & $S G$ & $E$ \\
\hline$\% \mathrm{~N}$-rendement & $\begin{array}{r}0,05 \\
0,36 \\
-0,41\end{array}$ & $\begin{array}{l}-0,57\left(^{*}\right) \\
- \\
-\end{array}$ & $\begin{array}{l}-0,59^{\star *} \\
-0,29 \\
-0,74^{* \star}\end{array}$ & $\begin{array}{l}-0,37^{\star} \\
-0,3 \\
-0,49^{\star}\end{array}$ & $\begin{array}{r}-0,02 \\
0,31 \\
-0,46\end{array}$ & $\begin{array}{l}-0,3 \\
-0,2 \\
-0,45\end{array}$ & $\begin{array}{l}0,48\left(^{*}\right) \\
0,42 \\
-\end{array}$ \\
\hline$\% \mathrm{~N}$-pelshenke & $\begin{array}{l}0,06 \\
0,01 \\
0,21\end{array}$ & $\begin{array}{l}-0,33 \\
- \\
-\end{array}$ & $\begin{array}{l}-0,17 \\
-0,14 \\
-0,23\end{array}$ & $\begin{array}{l}-0,15 \\
-0,19 \\
-0,08\end{array}$ & $\begin{array}{l}0,23 \\
0,28 \\
0,16\end{array}$ & $\begin{array}{l}0,54^{\star} \\
0,44 \\
0,75^{*}\end{array}$ & $\begin{array}{l}0,43 \\
0,6\left(^{*}\right) \\
-\end{array}$ \\
\hline$\% \mathrm{~N}$-dureté & $\begin{array}{l}-0,54^{\star *} \\
-0,69^{*} \\
-0,33\end{array}$ & $\begin{array}{l}0,16 \\
- \\
-\end{array}$ & $\begin{array}{l}-0,2 \\
-0,4 \\
-0,1\end{array}$ & $\begin{array}{l}-0,65^{\star \star \star} \\
-0,64^{\star} \\
-0,66^{\star \star}\end{array}$ & $\begin{array}{l}-0,34 \\
-0,51 \\
-0,03\end{array}$ & $\begin{array}{l}-0,64^{\star \star} \\
-0,68^{\star} \\
-0,55\left(^{*}\right)\end{array}$ & $\begin{array}{c}-0,17 \\
-0,18 \\
-\end{array}$ \\
\hline$\% N-W$ & $\begin{array}{l}0,2 \\
0,18 \\
0,26\end{array}$ & $\begin{array}{l}0,17 \\
- \\
-\end{array}$ & $\begin{array}{l}-0,1 \\
-0,24 \\
-0,01\end{array}$ & $\begin{array}{l}0,14 \\
0,03 \\
0,29\end{array}$ & $\begin{array}{l}0,36 \\
0,32 \\
0,41\end{array}$ & $\begin{array}{l}- \\
- \\
-\end{array}$ & $\begin{array}{l}0,68^{\star} \\
0,76^{*} \\
-\end{array}$ \\
\hline$\% N-P$ & $\begin{array}{l}-0,08 \\
-0,1 \\
-0,4\end{array}$ & $\begin{array}{l}0,15 \\
- \\
-\end{array}$ & $\begin{array}{r}-0,11 \\
-0,35 \\
0,12\end{array}$ & $\begin{array}{l}-0,14 \\
-0,22 \\
-0,03\end{array}$ & $\begin{array}{l}0,25 \\
0,2 \\
0,38\end{array}$ & $\begin{array}{l}- \\
-\end{array}$ & $\begin{array}{l}0,45\left(^{*}\right) \\
0,61\left(^{\star}\right) \\
-\end{array}$ \\
\hline$\% \mathrm{~N}-\mathrm{L}$ & $\begin{array}{l}0,48^{\star} \\
0,66^{\star} \\
0,8^{\star \star}\end{array}$ & $\begin{array}{l}0,41 \\
- \\
-\end{array}$ & $\begin{array}{l}0,39^{\star} \\
0,31 \\
0,49^{\star}\end{array}$ & $\begin{array}{l}0,57^{\star \star} \\
0,51\left(^{\star}\right) \\
0,69^{\star \star}\end{array}$ & $\begin{array}{l}0,26 \\
0,21 \\
0,4\end{array}$ & $\begin{array}{l}- \\
- \\
-\end{array}$ & $\begin{array}{l}0,55^{\star} \\
0,4 \\
-\end{array}$ \\
\hline
\end{tabular}

Pour chaque couple de variables, la première ligne correspond au coefficient de corrélation calculé pour l'ensemble des génotypes, la deuxième ligne à celui calculé pour les parents, et la troisième ligne à celui calculé pour les hybrides (les coefficients de corrélation n'ont été calculés que lorsqu'il y avait au moins 5 degrés de liberté). (") significatif au seuil de $10 \%$; " significatif au seuil de $5 \%$; ${ }^{* \star}$ significatif au seuil de $1 \% ;{ }^{* \star \star}$ significatif au seuil de $1 \%$. 
PELSHENKE

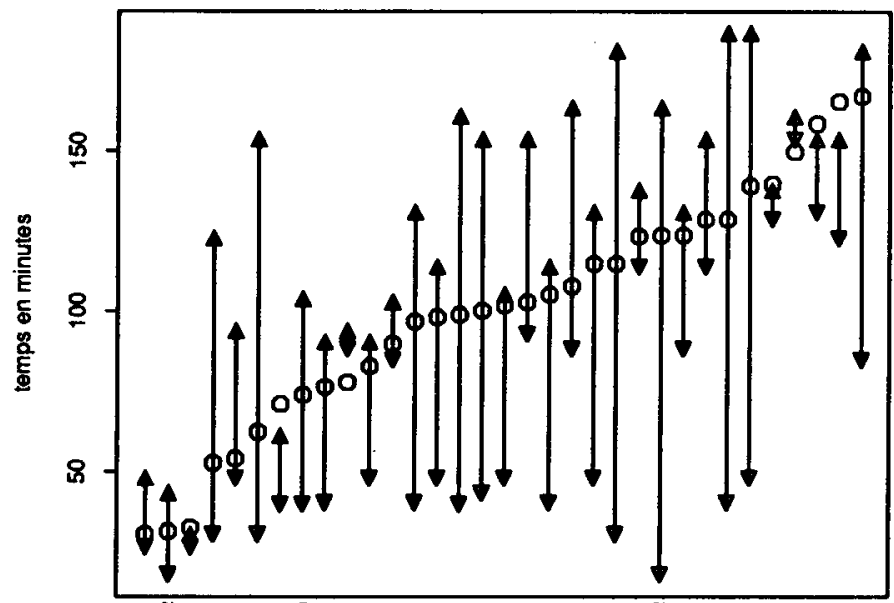

I⿳⺈⿴囗十大

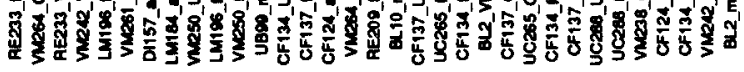

FORCE

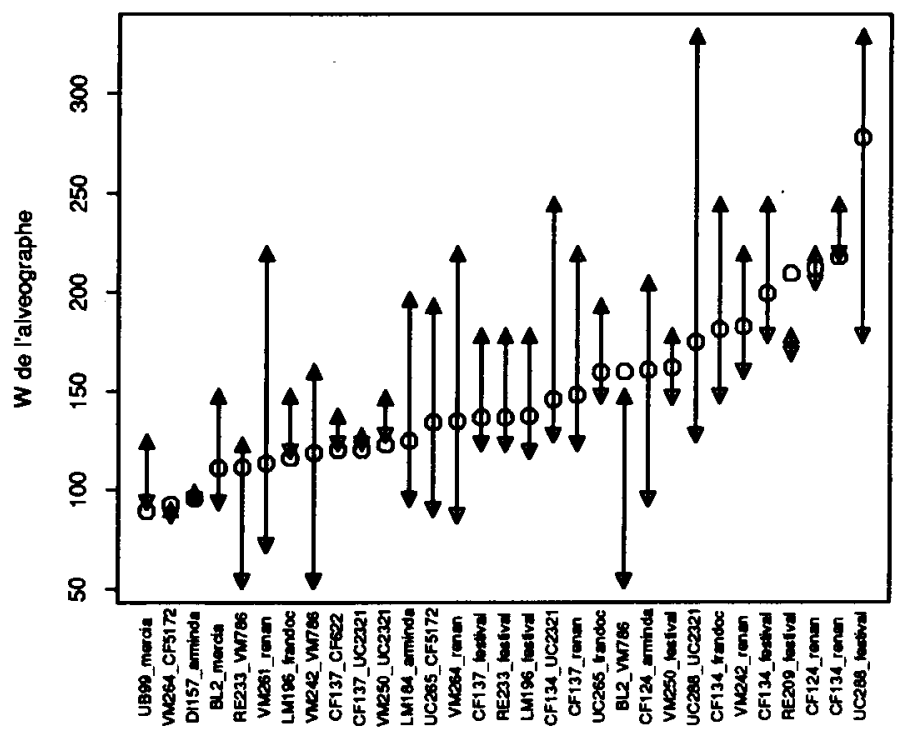

EXTENSIBILITE

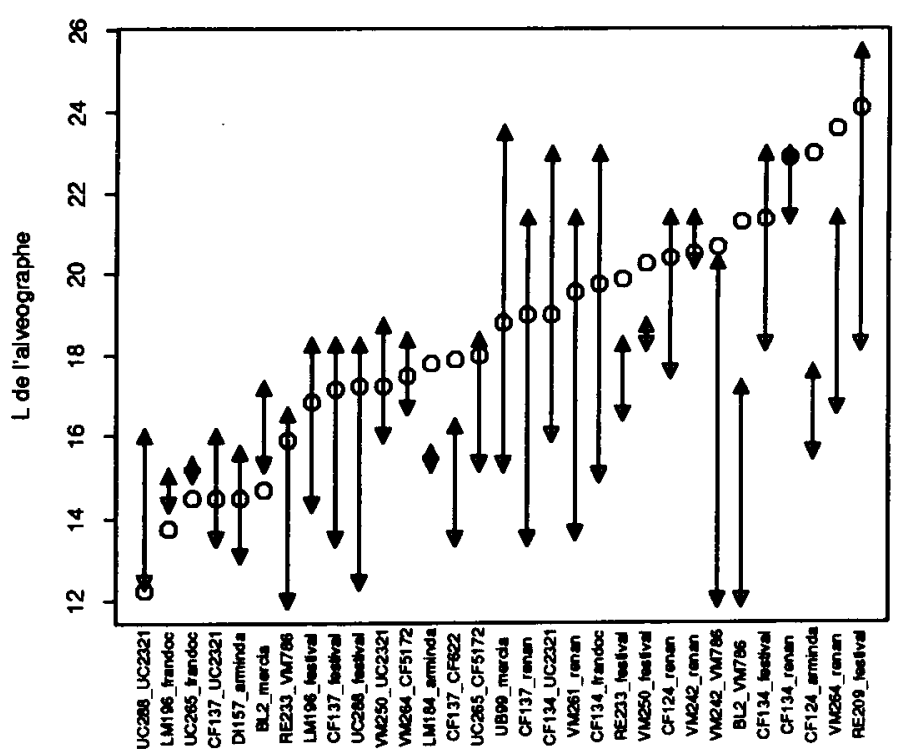

DURETE

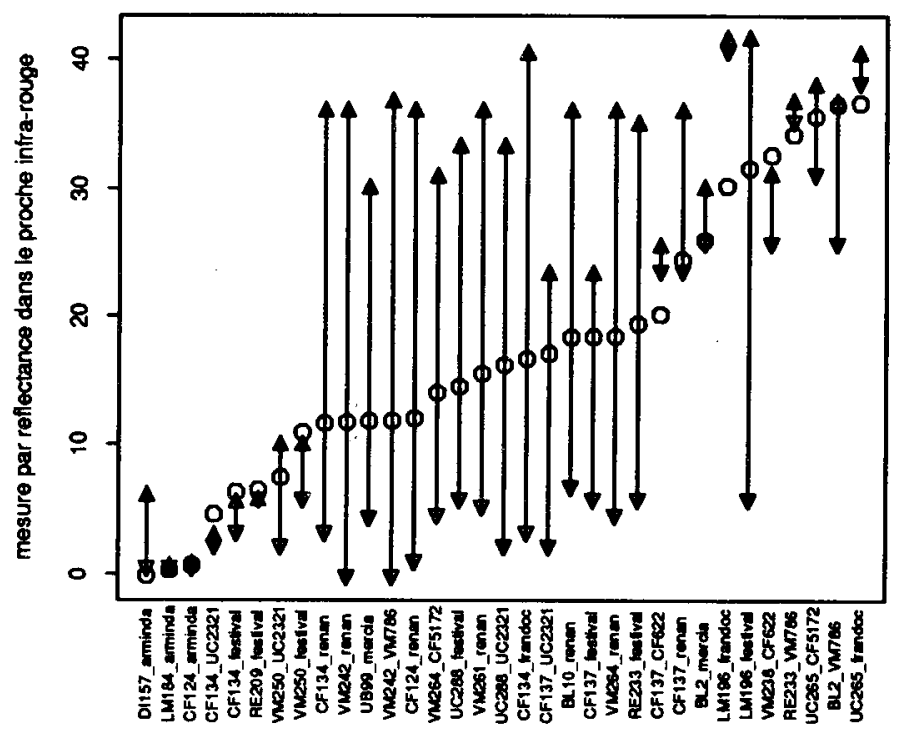

TENACITE

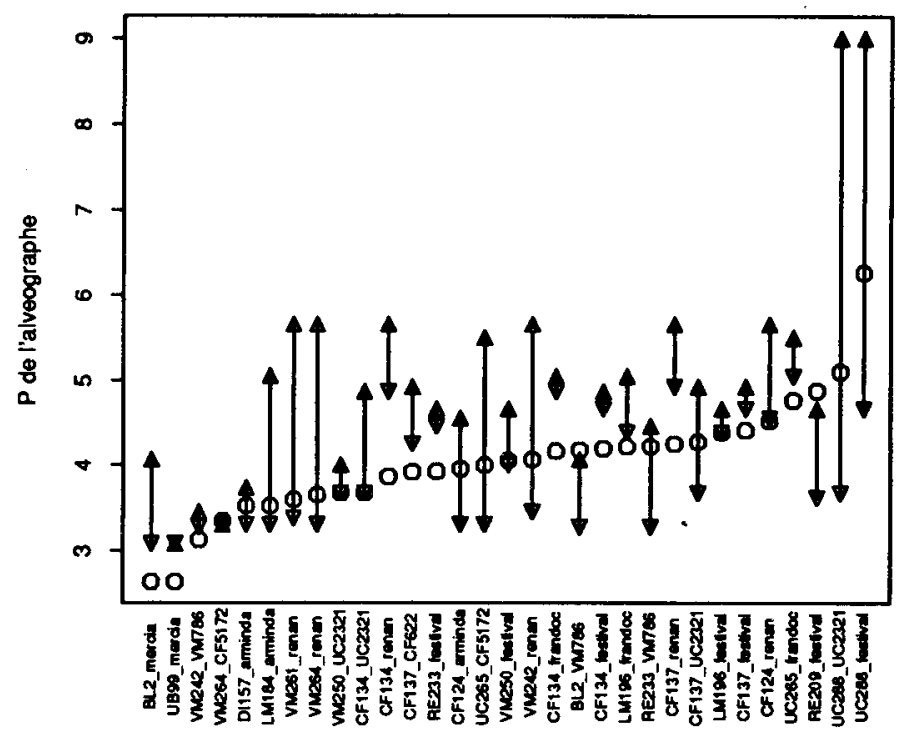

EQUILIBRE DE LA COURBE ALVEOGRAPHIQUE

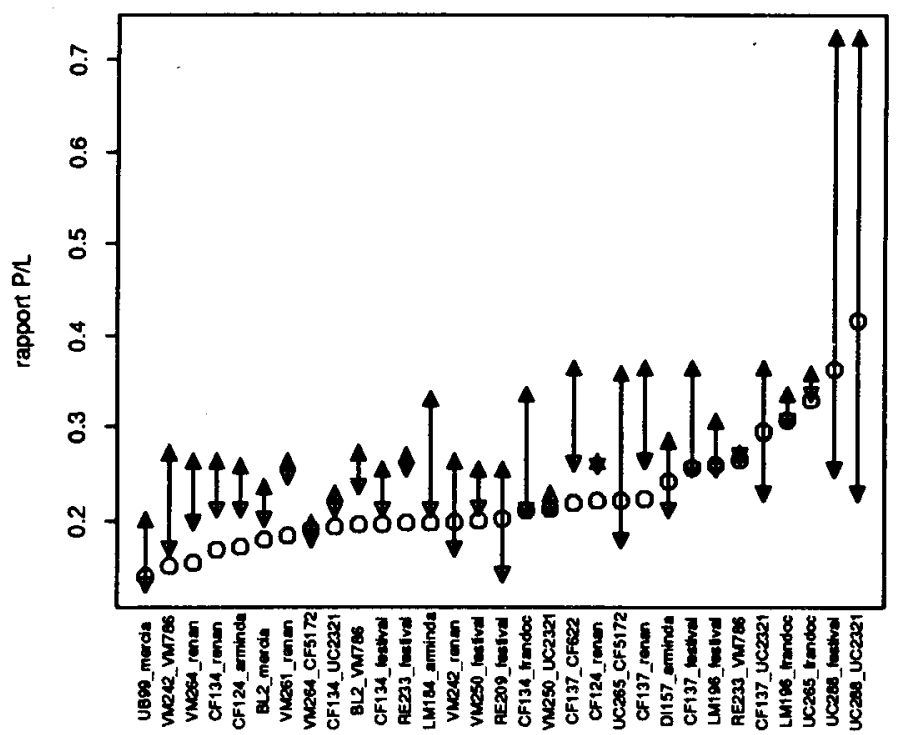


Tableau IV. Niveaux d'hétérosis (exprimés en \%) pour les 5 variables technologiques.

\begin{tabular}{lccccc}
\hline & Pelshenke & Dureté & $W$ & $P$ & $L$ \\
\hline Hétérosis PM & $+6,5^{\star}$ & -6 & -3 & $-8,9$ & $+7,7^{\star \star \star}$ \\
Hétérosis PS & $-19,3$ & $-32,2$ & $-19,4$ & $-17,9$ & -3 \\
\hline
\end{tabular}

À côté des valeurs d'hétérosis figurent les résultats du test de Student unilatéral permettant de tester la supériorité des hybrides par rapport aux parents moyen et supérieur : ${ }^{*}$ significatif au seuil de $5 \% ;{ }^{\star \star \star}$ significatif au seuil de $1 \%$.

dureté n'est pas encore parfaitement connu, mais ce caractère serait contrôlé par un gène majeur (peut-être 2), avec intervention de gènes mineurs (Symes, 1965, 1969 ; Mclntosh, 1988 ; Lukow et al, 1989 ; MacRitchie et al, 1990 ; Anjum et Walker, 1991). Le tableau IV et la figure 2 montrent que la dureté de l'hybride est intermédiaire par rapport à celle de ses parents, ce qui va dans le sens de ce déterminisme relativement simple et d'une additivité des effets des gènes impliqués dans les types "soft»et «medium hard».

Pour les industries de transformation, les types "medium hard" et "soft" présentent souvent des qualités antagonistes : par exemple, au niveau de la meunerie, un grain "medium hard" nécessite plus d'énergie pour être moulu qu'un grain «soft", mais le taux d'extraction de farine est plus faible pour le grain «soft» que pour le grain «medium hard". La production d'hybrides

Tableau V. Corrélations entre les 5 variables technologiques, calculées pour l'ensemble des génotypes, pour les parents et pour les hybrides.

Pelshenke Dureté Alvéographe

Dureté

$\begin{array}{ll}\text { Total } & 0,13 \\ \text { Parents } & 0,18\end{array}$

Hybrides $\quad 0,06$

W

$\begin{array}{llr}\text { Total } & 0,68^{\star \star \star} & -0,04 \\ \text { Parents } & 0,74^{\star \star *} & 0,01 \\ \text { Hybrides } & 0,58^{\star \star} & -0,14\end{array}$

$P$

$\begin{array}{llll}\text { Total } & 0,46^{\star \star \star} & 0,3^{\star} & 0,8^{\star \star \star} \\ \text { Parents } & 0,6^{\star \star} & 0,36\left(^{\star}\right) & 0,85^{\star \star \star} \\ \text { Hybrides } & 0,24 & 0,17 & 0,74^{\star \star \star}\end{array}$

$L$

$\begin{array}{lllll}\text { Total } & 0,16 & -0,35^{\star \star} & 0,29^{\star} & -0,19 \\ \text { Parents } & 0,19 & -0,36\left(^{\star}\right) & 0,25 & -0,21 \\ \text { Hybrides } & 0,11 & -0,33\left(^{\star}\right) & 0,37^{\star} & -0,1\end{array}$

(*) significatif au seuil de 10\%; * significatif au seuil de $5 \% ;{ }^{\star \star}$ significatif au seuil de $1 \%$; ${ }^{\star \star \star}$ significatif au seuil de $1 \%$.

Fig 2. Valeurs observées pour les hybrides (o), leurs parents inférieurs $(\boldsymbol{\nabla})$ et leurs parents supérieurs $(\boldsymbol{A})$, pour les 5 variables technologiques. Les triplets "hybrides + parents" sont disposés selon les valeurs croissantes des F1. 
"soft $x$ medium hard» pourrait permettre d'obtenir facilement un compromis intéressant. Cependant, la dureté intermédiaire obtenue dans le cas d'un croisement "soft x medium hard" est en fait la résultante de toute une gamme de duretés (les grains récoltés sur une $\mathrm{F} 1$ correspondent à une disjonction F2). Cette hétérogénéité serait sans doute à prendre en compte dans le cadre d'un processus de transformation industrielle.

Pour le Pelshenke l'hybride se situe entre le parent moyen et le parent supérieur (tableau IV et fig 2). Les relations entre le Pelshenke et les autres tests technologiques sont similaires pour les $\mathrm{F} 1$ et les lignées : on retrouve en particulier, chez les hybrides, une bonne corrélation entre les valeurs du Pelshenke et celles du W (tableau V), et la faible influence de la teneur en protéines sur ce test (tableau III). Ces 2 relations, déjà bien établies pour les lignées (Branlard et Dardevet, 1985 ; Khélifi et Branlard, 1992), sont d'ailleurs à l'origine de l'utilisation en sélection du Pelshenke, qui nécessite moins de grains et moins de temps de travail que l'alvéographe. Pour le $W$ de l'alvéographe, l'hybride se situe au niveau du parent moyen (tableau IV et fig 2). Ces résultats concernant le Pelshenke et le $\mathrm{W}$, indiquent que la force de la pâte est intermédiaire chez la F1. Ils vont dans le même sens que ceux obtenus par Borghi et al (1988), et que ceux présentés par Pickett (1993) dans sa synthèse bibliographique sur les travaux réalisés sur les blés hybrides.

La valeur intermédiaire pour le $W$ est obtenue avec une diminution de la ténacité et une augmentation de l'extensibilité. En effet, pour P, l'hybride est souvent proche du parent inférieur, alors que, pour L, l'hybride est proche du parent supérieur (tableau IV et fig 2). Ce résultat est analogue à celui obtenu par Perenzin et al (1992) sur un effectif d'hybrides comparable au nôtre. Cette situation conduit à des rapports $P / L$ plus faibles chez les F1, ce qui, dans le cas de notre échantillon parental composé majoritairement de blés peu tenaces, apparaît dommageable pour la qualité. En effet, les $P / L$ trop faibles (pâtes trop extensibles) sont défavorables (Dubois, 1988), au même titre que les $P / L$ trop élevés (pâtes trop tenaces manquant d'extensibilité).

Cependant, on assiste depuis quelques années à l'inscription d'un nombre croissant de blés «medium hard» (voire «hard"), alors qu'avant 1985 ces types de blés n'étaient pas cultivés de manière significative en France (Mahaut, 1993). Cela pose un problème pour la panification française, car ces blés sont généra- lement trop tenaces et pas assez extensibles. Le tableau $V$ montre qu'il y a effectivement une relation négative entre dureté et extensibilité, aussi bien pour les lignées que pour les hybrides. La production d'hybrides "soft x medium hard", en jouant favorablement sur l'extensibilité, pourrait permettre de valoriser ces nouvelles variétés «medium hard» tout en préservant un bon équilibre de la courbe alvéographique.

Le tableau III montre qu'il n'y a pas de relation entre le $\mathrm{W}$ ou le $\mathrm{P}$ de l'alvéographe, et la teneur en protéines. En revanche cette dernière est corrélée positivement avec l'extensibilité. Cette corrélation a déjà été signalée, aussi bien pour des lignées (Branlard et Dardevet, 1985), que pour des hybrides (Perenzin et al, 1992).

\section{CONCLUSION}

Notre étude montre que la sélection de blés hybrides de bonne qualité ne devrait pas poser de problèmes majeurs. En effet, pour les 3 tests technologiques que nous avons utilisés, la $F 1$ se situe entre ses 2 parents. En choisissant des parents de bonne qualité, on devrait donc obtenir une $\mathrm{F} 1$ ayant une valeur technologique intéressante, tout en bénéficiant de l'hétérosis pour le rendement en grains.

\section{RÉFÉRENCES}

Anjum FM, Walker CE (1991) Review on the significance of starch and protein to wheat kernel hardness. J Sci Food Agric 56, 1-13

Becker RA, Chambers JM, Wilks AR (1988) The New $S$ Language: a programming environment for data analysis and graphics. Wadsworth \& Brooks/Cole, Pacific Grove, California, 702 p

Bingham J, Lupton FGH (1987) Production of new varieties: an integrated research approach to plant breeding. In : Wheat breeding, its scientific basis (FGH Lupton, ed), Chapman \& Hall, Londres et New York, 487-538

Boland OW, Walcott JJ (1985) Levels of heterosis for yield and quality in a F1 hybrid wheat. Aust $J$ Agric Res 36, 545-552

Borghi B, Perenzin M, Nash RJ (1988) Agronomic and qualitative characteristics of ten bread wheat hybrids produced using a chemical hybridizing agent. Euphytica 39, 185-194

Borghi B, Perenzin M, Nash RJ (1989) Combining ability estimates in bread wheat and performance of 100 hybrids produced using a chemical hybridizing agent. J Genet Breed 43, 11-16 
Branlard G, Dardevet M (1985) Diversity of grain proteins and bread wheat quality. I. Correlation between gliadin bands and flour quality characteristics. J Cereal Sci 3, 329-343

Brears T, Hydon AG, Bingham J (1988) An assessment of the feasibility of producing $F 1$ and $F 2$ hybrids for the UK. In : Proc 7th Int Wheat Genet Symp, 1057-1062

Brunori A, Galterio G, Zannettino C, Pogna NE (1989) Bread-making quality indices in Triticum aestivum progenies. Implications in breeding for better bread wheat. Plant Breeding 102, 222-231

Carrillo JM, Rousset M, Qualset CO, Kasarda DD (1990) Use of recombinant inbred lines of wheat for study of associations of high-molecular weight glutenin subunit alleles to quantitative traits: 1) grain yield and quality prediction tests. Theor Appl Genet $79,321-330$

Dubois $M$ (1988) Contribution de la rhéologie empirique à la détermination de la qualité des blés et des farines dans le monde : l'alvéographe Chopin. Industrie des céréales 53, 15-25

Khélifi D, Branlard G (1992) The effects of HMW and LMW subunits of glutenin and of gliadins on the technological quality of progeny from four crosses between poor breadmaking quality and strong wheat cultivars. J Cereal Sci 16, 195-209

Lukow OM, Mac Kenzie RIH, De Pauw RM (1989) Genetic implications of kernel hardness variation in canada prairie spring wheats. Can J Plant Sci 69, 667-674

McIntosh RA (1988) Catalogue of gene symbols for wheat. In : Proc 7th Int Wheat Genet Symp, 12251323

MacRitchie F, du Cros DL, Wrigley CW (1990) Flour polypeptides related to wheat quality. Adv Cereal Sci Technol X, 79-14
Mahaut B (1993) Dureté des blés : méthodes de mesure; situation des blés français. Industrie des Céréales, 39-44

Morgan CL, Austin RB, Ford MA, Bingham J, Angus WJ, Chowdhury $S$ (1989) An evaluation of F1 hybrid winter wheat genotypes produced using a chemical hybridizing agent. J Agric Sci Cam 112, 143-149

Oury FX (1990) Une contribution à la sélection simultanée sur plusieurs caractères : l'utilisation de la programmation linéaire. agronomie 10, 517-524

Oury FX, Brabant P, Pluchard P, Bérard P, Rousset M (1990) Étude multilocale de blés hybrides : niveaux d'hétérosis et élaboration du rendement. agronomie 10, 735-748

Oury FX, Brabant $P$, Pluchard $P$, Bérard $P$, Rousset $M$ (1993) Une étude sur la supériorité des blés hybrides au niveau des capacités de remplissage du grain : résultats d'une expérimentation multilocale. agronomie 13, 381-393

Payne PI, Nightingale MA, Krattiger AF, Holt LM (1987) The relationship between HMW glutenin subunit composition and the bread-making quality of British-grown wheat varieties. J Sci Food Agric 40, 51-65

Perenzin M, Pogna NE, Borghi B (1992) Combining ability for breadmaking quality in wheat. Can J Plant Sci 72, 743-754

Pickett AA (1993) Hybrid wheat: results and problems. Paul Parey Scientific Publishers, Berlin et Hambourg, $259 p$

Symes KJ (1965) The inheritance of grain hardness in wheat as measured by the particle size index. Aust $J$ Agric Res 16, 113-123

Symes KJ (1969) Influence of a gene causing hardness on the milling and baking quality of two wheats. Aust J Agric Res 20, 971-979 